\title{
Mathematical Modelling of the COVID-19 Epidemic in Northern Ireland in 2020
}

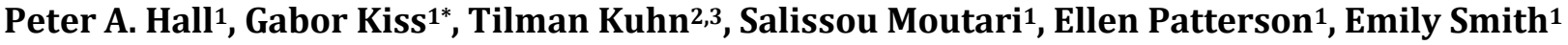 \\ ${ }^{1}$ Mathematical Science Research Centre, School of Mathematics \& Physics, Queen's University Belfast, \\ Belfast, Northern Ireland, United Kingdom \\ ${ }^{2}$ School of Biological Sciences, Queen's University Belfast, Belfast, Northern Ireland, United Kingdom \\ ${ }^{3}$ Heidelberg Institute of Global Health (HIGH), Heidelberg University Hospital, Heidelberg, Germany \\ Email: *g.kiss@qub.ac.uk
}

How to cite this paper: Hall, P.A., Kiss, G., Kuhn, T., Moutari, S., Patterson, E. and Smith, E. (2021) Mathematical Modelling of the COVID-19 Epidemic in Northern Ireland in 2020. Open Journal of Modelling and Simulation, 9, 91-110.

https://doi.org/10.4236/ojmsi.2021.92006

Received: February 27, 2021

Accepted: April 9, 2021

Published: April 12, 2021

Copyright (c) 2021 by author(s) and Scientific Research Publishing Inc. This work is licensed under the Creative Commons Attribution International License (CC BY 4.0).

http://creativecommons.org/licenses/by/4.0/ Open Access

\begin{abstract}
In this study, we investigate the dynamics of the COVID-19 epidemic in Northern Ireland from $1^{\text {st }}$ March 2020 up to $25^{\text {th }}$ December 2020, using several copies of a Susceptible-Exposed-Infectious-Recovered (SEIR) compartmental model, and compare it to a detailed publicly available dataset. We split the data into 10 time intervals and fit the models on the consecutive intervals to the cumulative number of confirmed positive cases on each interval. Using the fitted parameter estimates, we also provide estimates of the reproduction number. We also discuss the limitations and possible extensions of the employed model.
\end{abstract}

\section{Keywords}

Pandemic, Epidemic, SARS-CoV-2, COVID-19, Compartmental Model, SEIR Model, Basic Reproduction Number, Effective Reproduction Number, Parameter Estimates, Fitted Model

\section{Introduction}

The coronavirus disease 2019 (COVID-19), caused by the severe acute respiratory syndrome coronavirus 2 (SARS-CoV-2), was early reported in China mainland. In January 2020, the World Health Organization declared the outbreak a Public Health Emergency of International Concern [1], and a pandemic in March 2020 [2]. At the time of this study, the number of confirmed cases was over 80 million, and the disease caused more than 1.8 million deaths worldwide [3]. Since the early signs of the pandemic, despite the challenges posed by this unknown disease, see [4], several mathematical and statistical models of varying complex- 
ity have been built and used to predict the potential future development of the pandemic in many countries including China [5], United States [6], United Kingdom [7], Italy [8] [9], Spain [10], Germany [11], France [12], Ireland [13], Hungary [14], and Mexico [15]. Such models must be considered in local context taking into account the social, cultural, demographic, economic and other granular issues that differ between populations yet may dramatically influence the dynamics of any epidemic [16] [17].

The COVID-19 pandemic reached England around late January 2020, and about one month later, it was present in all the four regions of the United Kingdom (UK). The epidemic reached its first peak in the UK in April 2020, and since then various lockdown measures have been introduced and eased. The rationale behind these lockdown measures is to reduce the chances of introducing new infections from outside, to control the spread of the disease domestically and to reduce the pressure on the National Health Service. In the UK, although there is a nation-wide effort to mitigate the challenges posed by COVID-19, major public services affected by the pandemic, including public health services and education, are the responsibility of the devolved administrations, namely England, Scotland, Wales and Northern Ireland. This results in some variations in terms of public health policies, for example, the timing of the launch of test-andtrace strategies and the details of lockdown. This heterogeneity is confirmed not only by the distribution of the levels of the infection across the four regions of the UK but also by their own different roadmap for easing lockdown restrictions after the first wave of the pandemic. For example, England and Northern Ireland moved faster to open some businesses including non-essential retails and restaurants before Wales and Scotland.

Our goal is to take advantage of the detailed publicly available datasets and to capture the history of the pandemic in Northern Ireland by fitting a compartmental model to empirical data. More specifically, we estimate the parameters of a system of ordinary differential equations so that its numerical solution for the cumulative positive cases will reflect the daily reported cumulative number of positive tests. The corresponding numerical solutions enable us not only to estimate the historical number of susceptible, exposed, infectious and recovered individuals but also to make some predictions of these numbers. Hence, the fitted model can be used to monitor and assess the state of the epidemic, its future evolution as well as the impact of specified levels of intervention measures. To the best of our knowledge, this is the first research work assessing the course of the pandemic in Northern Ireland using tools from mathematical epidemiology.

\section{Materials and Methods}

\subsection{Data}

In Northern Ireland (NI), the Department of Health (DoH) publishes daily updates of COVID-19 related data [18]. Although the first reported case of COVID-19 in NI dated from $11^{\text {th }}$ January 2020 , there are no other confirmed cases until $5^{\text {th }}$ 
March 2020. Hence, this study focuses on data from $1^{\text {st }}$ March 2020 up to $25^{\text {th }}$ December 2020. The rationale behind the choice of this time frame is that 1) either the case reported on $11^{\text {th }}$ January 2020 had no effect on the course of the epidemic; 2) or there were some individuals, whose number will be estimated by the method describe in Section 3, already carrying the disease without symptoms hence being undetected before $1^{\text {st }}$ March 2020; 3) new lockdown measures had been introduced on $26^{\text {th }}$ December 2020 ; 4) data collection was less consistent between $26^{\text {th }}$ December 2020 and $31^{\text {st }}$ December 2020 5) no information is currently available on the effect of the new strains first detected in November 2020.

This study is based on data sets from the aforementioned period, and in particular, the time series of the daily number of confirmed cases i.e., positive tests or the daily incidence of infection, Figure 1. However, the basic model, described below, captures the number of infected at any given time $t \geq 0$, i.e., the prevalence of infection. To overcome this issue, we generated the number of cumulative cases from our data set, and extended the basic model so that it provides the estimation of the cumulative cases at any given time $t \geq 0$. Figure 2 depicts the cumulative number of confirmed cases in Northern Ireland from $1^{\text {st }}$ March 2020 to $25^{\text {th }}$ December 2020.

Furthermore, we divide the period from $1^{\text {st }}$ March 2020 to $25^{\text {th }}$ December 2020 into 10 overlapping intervals $T_{i}, i=0, \cdots, 9$, such that

- the first 9 intervals, $T_{i}=\left[t_{i, l}, t_{i, r}\right], i=0, \cdots, 8$ with $t_{i+1, l}=t_{i, r}$, have an equal length of 30 days; and

- the last interval, $T_{9}=\left[t_{9, l}, t_{9, r}\right]$ with $t_{9, l}=t_{8, r}$, has a length of 39 days.

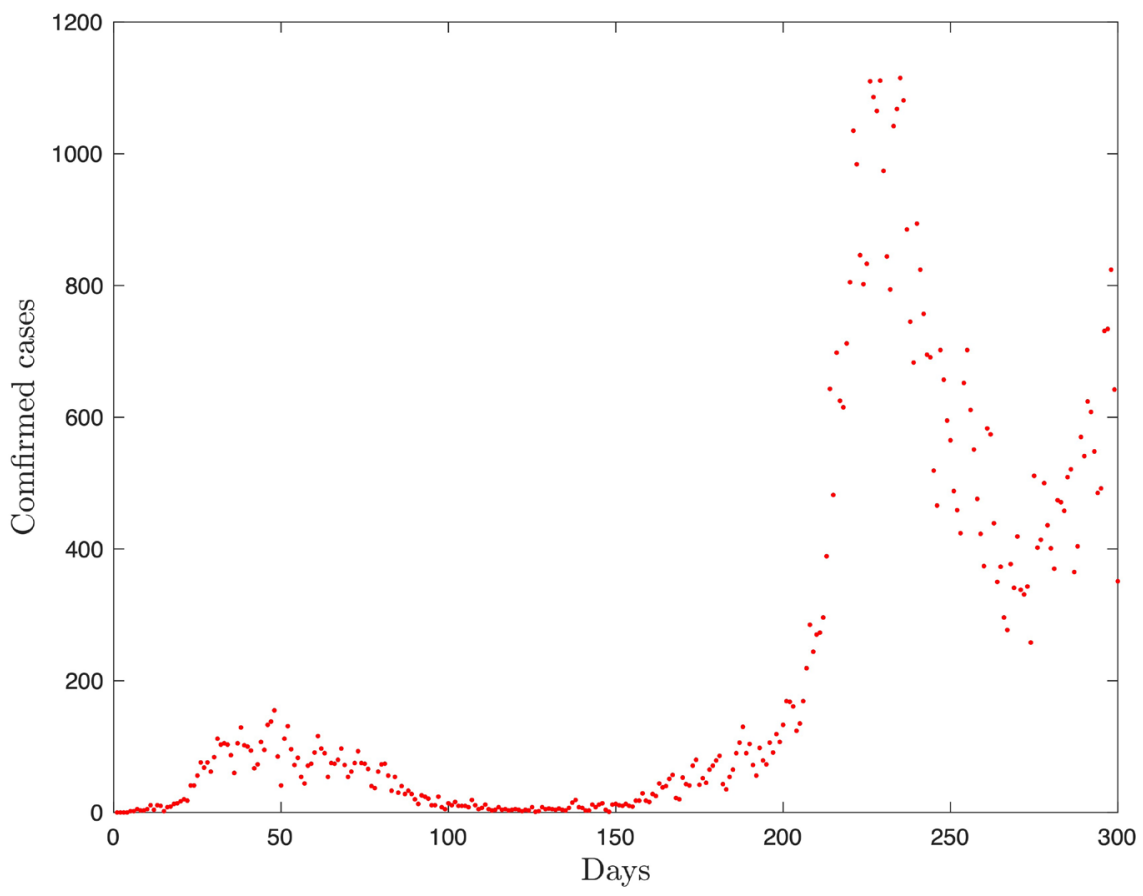

Figure 1. Daily incidence of infection in Northern Ireland from $1^{\text {st }}$ March 2020 to $25^{\text {th }}$ December 2020 from [18]. 


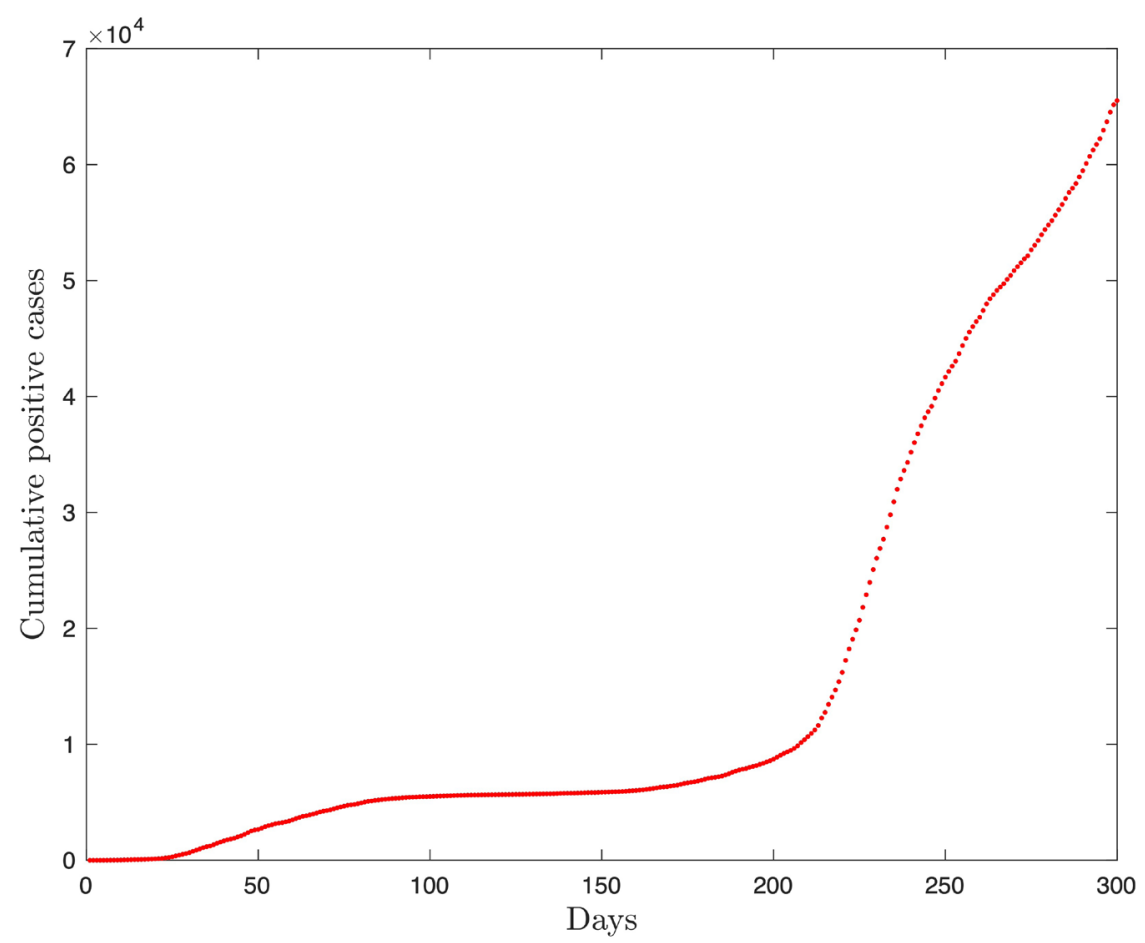

Figure 2. Cumulative number of reported positive tests in Northern Ireland from $1^{\text {st }}$ March 2020 to $25^{\text {th }}$ December 2020.

As such, the first interval includes the period before the first nationwide lockdown was introduce in the UK on 27th March 2020. We let $D_{i}$ denote the data from the $i^{\text {th }}$ interval $T_{i}$. For the purpose of this study, the population size of Northern Ireland, $N$, was kept at the estimated value of $1,893,700$ [19] with no incoming or outgoing travel.

\subsection{The Transmission Model}

Compartmental models have been used widely to model the dynamics of various infectious diseases [20] [21]. The most commonly used mathematical descriptions in modelling the early phase of the outbreak of COVID-19 are variants of the SIR model derived in [22]. The SIR model assumes infectiousness immediately after the exposure to the causative agent. However, early studies on epidemiological and clinical features of COVID-19 identified a significant incubation period of 6 days on average, see e.g. [23] [24]. Therefore, in this study, we use different SEIR models on the consecutive time intervals to approximate the course of the pandemic in Northern Ireland in 2020. This approach enables us to model the number of exposed, infected but not yet infectious, individuals in the population. It also enables to capture the continuous growth in the number of new cases when strict intervention measures reduce drastically the contact rate (or the transmission probability). This model does not include several other possible features such as asymptomatic infections, the presence of variants of the virus or vaccination.

An SEIR model has four non-negative state variables, which are described in 
Table 1, and the corresponding flow of the disease transmission is depicted in Figure 3.

The disease is transmitted, at a rate $\beta>0$, from an infectious individual to a susceptible one, who then becomes exposed. Individuals from compartment $E$ move to compartment $I$ at a rate $a>0$, where $\frac{1}{a}$ is the average incubation period. Subjects from compartment $I$ progress to compartment $R$ at a rate $\gamma>0$, where $\frac{1}{\gamma}$ is the average duration of infection. Furthermore, we assume that the population size $N$ is independent of time, and the exposure to the pathogen offers immunity for the time period of the study. Notice that the model is without vital dynamics, that is we do not consider the effect of birth and death processes, hence $N=S+E+I+R$. Thus, the described flow of the disease transmission results in

$$
\begin{aligned}
& \dot{S}=-\frac{\beta S I}{N} \\
& \dot{E}=\frac{\beta S I}{N}-a E \\
& \dot{I}=a E-\gamma I \\
& \dot{R}=\gamma I
\end{aligned}
$$

To approximate the dynamics of COVID-19 in NI in 2020, we use several copies of the model (1) with an additional non-negative variable $I_{c}(t)$. As mentioned in Section 0, the DoH provides the daily number of new COVID-19 cases; however, the model (1) does not include a variable for such data. Therefore, we introduce the non-negative variable $I_{c, i}(t)$, which represents the cumulative number of infected individuals in the population at any time $t$ in the interval $T_{i}, i=0, \cdots, 9$. The variable $I_{c, i}(t)$ does not affect the transmission dynamics; its role is to capture the cumulative number of COVID-19 cases.

To describe the dynamics of the outbreak, in each time interval $T_{i}=\left[t_{i, l}, t_{i, r}\right], i=0, \cdots, 9$, we consider the following model:

Table 1. State variables.

\begin{tabular}{cc}
\hline Variable & Meaning \\
\hline$S(t)$ & The number of susceptible individuals at time $t$ in the population. \\
$E(t)$ & The number of exposed individuals at time $t$ in the population. \\
$I(t)$ & The number of recovered individuals at time $t$ in the population. \\
\hline$R(t)$ & $\frac{\beta S I}{N}$
\end{tabular}

Figure 3. Transmission diagram for the SEIR model. 


$$
\begin{aligned}
& \dot{S}_{i}=-\frac{\beta_{i} S_{i} I_{i}}{N} \\
& \dot{E}_{i}=\frac{\beta_{i} S_{i} I_{i}}{N}-a_{i} E_{i} \\
& \dot{I}_{i}=a_{i} E_{i}-\gamma_{i} I_{i} \\
& \dot{R}_{i}=\gamma_{i} I_{i} \\
& \dot{I}_{c, i}=a_{i} E_{i}
\end{aligned}
$$

with the following initial conditions

$$
\begin{aligned}
& \left(S_{i}^{0}, E_{i}^{0}, I_{i}^{0}, R_{i}^{0}, I_{c, i}^{0}\right) \\
& = \begin{cases}(N-2,2,0,0,0), & i=0, \\
\left(S_{i-1}\left(t_{i-1, r}\right), E_{i-1}\left(t_{i-1, r}\right), I_{i-1}\left(t_{i-1, r}\right), R_{i-1}\left(t_{i-1, r}\right), I_{c, i-1}\left(t_{i-1, r}\right)\right), & 1 \leq i \leq 9 .\end{cases}
\end{aligned}
$$

Moreover, we assume that $N=S_{i}+E_{i}+I_{i}+R_{i}$ is independent of $i$, and the parameters of the model reflect introduced or relaxed measures instantaneously i.e., in contrast with the formulations in [25] [26], we do not assume the exponential decay from one parameter value to the other. The latter assumption is motivated by the implementation of COVID-19 related interventions in NI without any transition period; although we recognise that this is an approximation of the real world behaviour. The transitions between the time intervals, $T_{i}$, are arbitrary and do not necessarily match the introduction and release of control measures.

\subsection{Basic Reproduction Number}

The basic reproduction number, $\mathcal{R}_{0}$, is the most commonly used metric to characterize the early phase of a disease outbreak. It quantifies the transmissibility of the disease, and it can be defined as the mean number of secondary infections induced by an infectious individual in a completely susceptible population. In general, if $\mathcal{R}_{0}>1$ an epidemic occurs, and the larger the values of $\mathcal{R}_{0}$ the more challenging to bring the outbreak under control. On the other hand, if $\mathcal{R}_{0} \leq 1$, no epidemic occurs and the disease dies out. For the model (1), it can be shown that

$$
\mathcal{R}_{0}=\frac{\beta}{\gamma}
$$

is a threshold number ${ }^{1}$, see [28]. Furthermore, when $\mathcal{R}_{0}>1$, i.e. the occurrence of an outbreak, the system tends to a unique endemic equilibrium.

For the model (2), we define

$$
\mathcal{R}_{0, i}=\frac{\beta_{i}}{\gamma_{i}} .
$$

However, since we assume complete immunity, then in the subsequent time intervals the population is not fully susceptible anymore. Therefore, in Section 12, we provide the so-called effective reproduction number defined as

${ }^{1}$ Although the formulae for $\mathcal{R}_{0}$ of an $S I R$ model and an SEIR model are the same, i.e. they have the same epidemiological interpretations, in the presence of incubation time, an SIR model underestimates the reproductive number [27]. 


$$
\mathcal{R}_{t, i}=\frac{S(t)}{N} \mathcal{R}_{0, i} .
$$

Note that, by using the data, $\frac{S(t)}{N} \approx 0.9734$ for $t=t_{9, r}$.

\subsection{Parameter Fitting}

To fit the model (2) to the data, we fit the model variables $I_{c, i}$ to the data $D_{i}$, from the $I^{\text {th }}$ interval $T_{i}$, as follows. For each time interval $T_{i}, i=0, \cdots, 9$, numerical solutions of the model (2) with initial condition (3) are generated while the corresponding nonlinear curve-fitting problem was solved in the least-squares sense. The ranges of these non-negative parameters $\beta_{i}, a_{i}$ and $\gamma_{i}$, used in the fitting process, are given in Table 2 . The choice of the interval of the average incubation period and recovery time (or the period of communicability) is consistent with other recent reports [23] [24] [29] [30] [31]. Also, the chosen upper bounds allow the algorithm to search parameter values in a relatively large range. The fitting process for each time interval $T_{i}$ was initiated with $\beta_{i}=1 / 5, a_{i}=1 / 8$ and $\gamma_{i}=19 / 100$ for $i=0, \cdots, 9$. In addition, for $i=0$, we used $E_{0}^{0} \in[1 e-8,1 e 2]$ to estimate the number of initially exposed yet not infectious to generate the epidemic in NI. All the computations were made using Matlab [32] leveraging the functions ode23 and lsqcurvefit.

\section{Result}

Using our model, we present numerical solutions for the model (2) as well as the corresponding fitted parameters. Furthermore, we compare our estimates of $\mathcal{R}_{0}$ with the estimates provided by the DoH [33].

\subsection{Early Spread of the Disease Pre-Lockdown Period}

The DoH reported one positive test on $11^{\text {th }}$ January 2020 . However, there were no additional cases reported until $5^{\text {th }}$ March 2020 when two positive tests were reported, and from this date onwards, the number of new cases were at least two. We started our data-fitting with parameters $\beta_{0}, a_{0}$ and $\gamma_{0}$ as of $1^{\text {st }}$ March 2020 , and we also fit $E_{0}^{0}$, the number of exposed at $t=0$; the initial guess for $E_{0}^{0}$ was 2.

The first interval, $T_{0}$, ends on $30^{\text {th }}$ March 2020, i.e., three days after the UK nationwide lockdown measures were introduced. After fitting the model, the estimated number of exposed individuals in this time interval, denoted $\tilde{E}_{0}^{0}$, is about

Table 2. Ranges of the parameters in the model (2), for $i=0, \cdots, 9$.

\begin{tabular}{ccc}
\hline Parameter & Meaning & Range \\
\hline$\beta_{i}$ & Transmission rate & $0-1$ \\
$a_{i}^{-1}$ & Average incubation period (days) & $2-20$ \\
$\gamma_{i}^{-1}$ & Average infectious period (days) & $2-20$ \\
\hline
\end{tabular}


7.238, and the values of the corresponding fitted parameters are presented in the first row in Table 3. The component $I_{c, 0}$ of the numerical solution of the model (2) in $T_{0}$, using the initial condition $\left(S_{0}^{0}, E_{0}^{0}, I_{0}^{0}, R_{0}^{0}, I_{c, 0}^{0}\right)=\left(N-\tilde{E}_{0}^{0}, \tilde{E}_{0}^{0}, 0,0,0\right)$ and parameters from Table 3, is presented in Figure 4 along with the number of positive cases, $D_{0}$. Figure 5 shows components $E, I$ and $R$ for the numerical solutions of the model (2). In Figure 6, we present the course of the epidemic without lockdown (or any intervention), which results from computing the solution of (2) on $T_{0}$ using the parameters and the initial condition described above.

Table 3. Model parameter estimates.

\begin{tabular}{cccccc}
\hline$i$ & Days & $\tilde{\beta}_{i}$ & $\tilde{a}_{i}$ & $\tilde{\gamma}_{i}$ & $\mathcal{R}_{0, i}$ \\
\hline 0 & $1-30$ & 0.5607 & 0.2902 & 0.1898 & 2.9539 \\
1 & $30-59$ & 0.4842 & 0.2058 & 0.5000 & 0.9684 \\
3 & $59-88$ & 0.4344 & 0.1880 & 0.5000 & 0.8688 \\
4 & $88-117$ & 0.1999 & 0.0500 & 0.3803 & 0.5255 \\
5 & $117-146$ & 0.0215 & 0.0500 & 0.0500 & 0.4297 \\
6 & $146-175$ & 0.1487 & 0.2543 & 0.0500 & 2.9742 \\
7 & $175-204$ & 0.1015 & 0.2020 & 0.0500 & 2.0298 \\
8 & $204-233$ & 0.4950 & 0.1456 & 0.2973 & 1.6649 \\
9 & $233-262$ & 0.3377 & 0.0759 & 0.5000 & 0.6753 \\
\hline
\end{tabular}

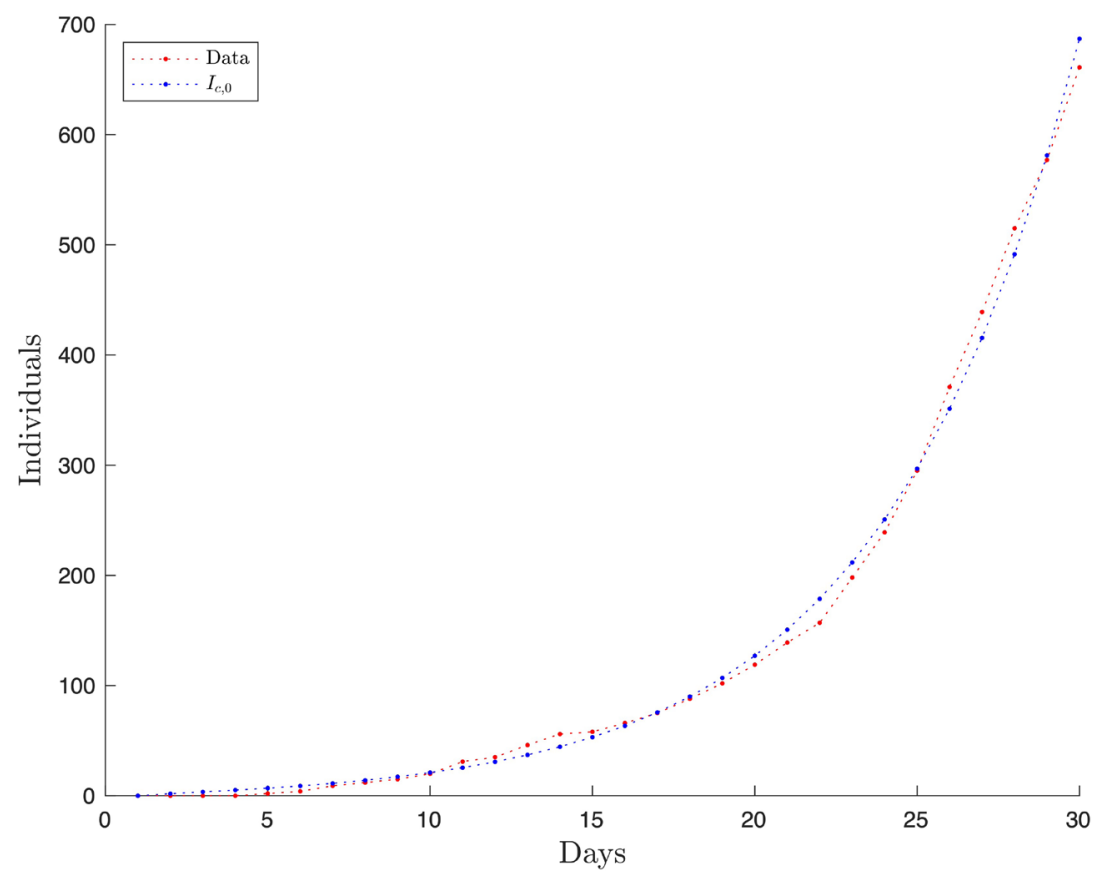

Figure 4. The blue dotted curve is the $I_{c, 0}$ component of the numerical solution of (2) with $\left(S_{0}^{0}, E_{0}^{0}, I_{0}^{0}, R_{0}^{0}, I_{c, 0}^{0}\right)=\left(N-\tilde{E}_{0}^{0}, \tilde{E}_{0}^{0}, 0,0,0\right)$ and parameters from Table $3, \quad i=0$. The red dotted curve represents $D_{0}$. 


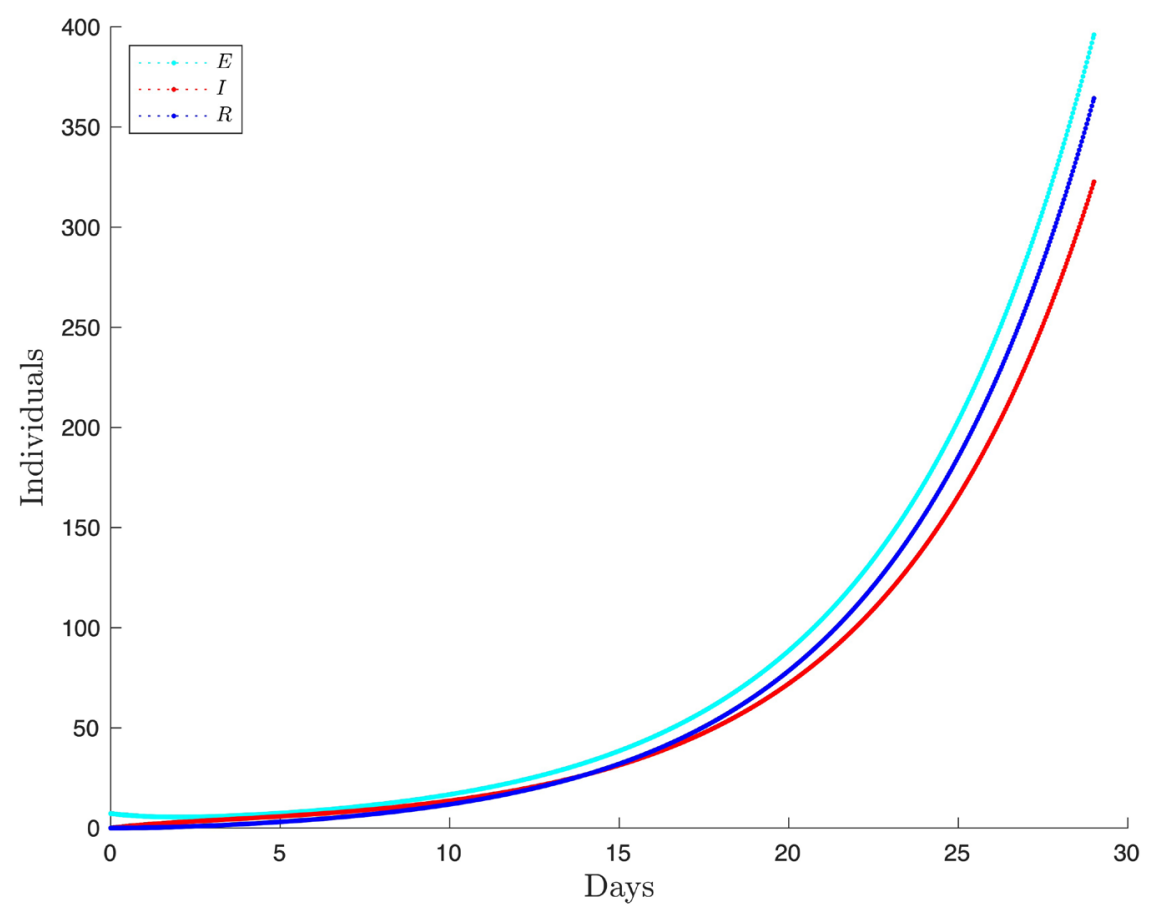

Figure 5. Compartments $E, I$ and $R$ of the numerical solution of (2) with $\left(S_{0}^{0}, E_{0}^{0}, I_{0}^{0}, R_{0}^{0}, I_{c, 0}^{0}\right)=\left(N-\tilde{E}_{0}^{0}, \tilde{E}_{0}^{0}, 0,0,0\right)$ and parameters from Table $3, \quad i=0$.

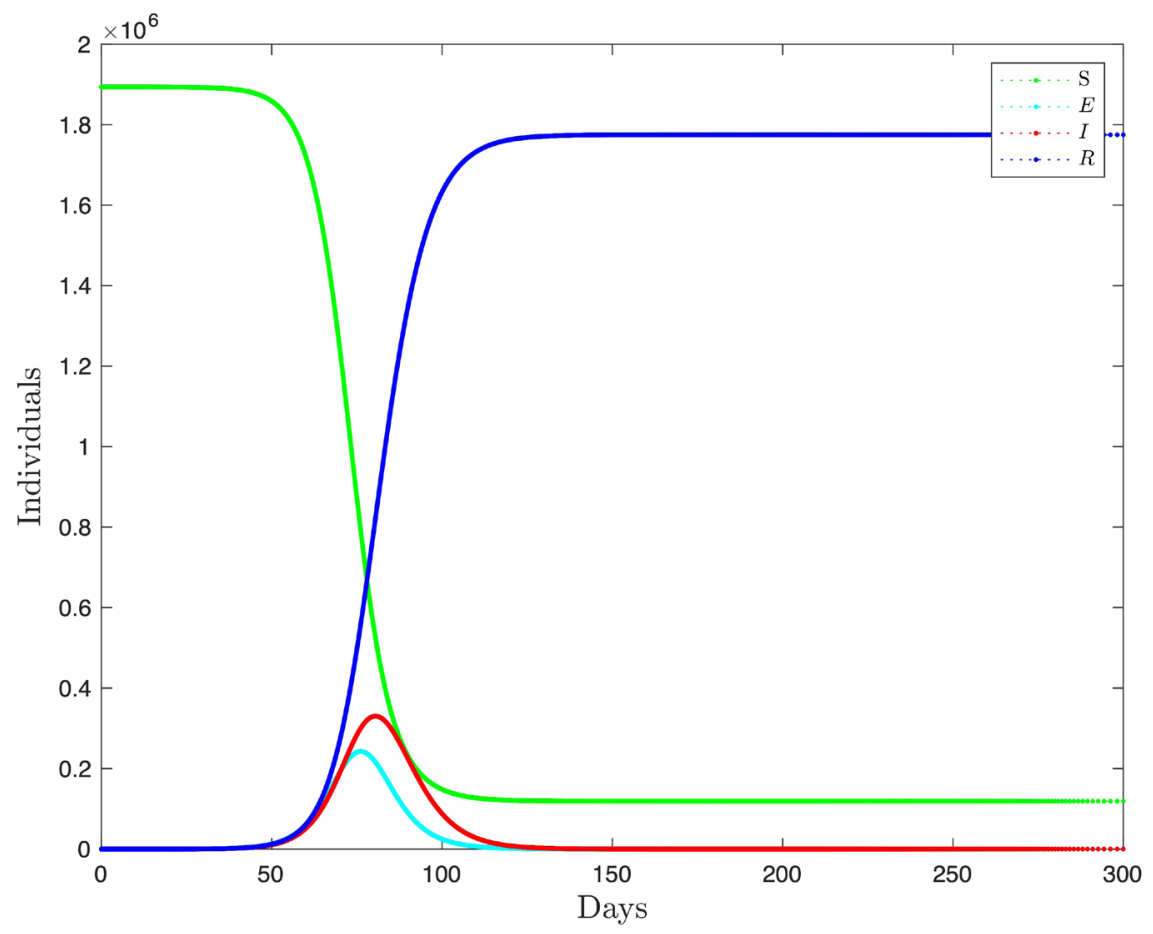

Figure 6. Numerical solution of (2) with $\left(S_{0}^{0}, E_{0}^{0}, I_{0}^{0}, R_{0}^{0}, I_{c, 0}^{0}\right)=\left(N-\tilde{E}_{0}^{0}, \tilde{E}_{0}^{0}, 0,0,0\right)$ and parameters from Table 3, $i=0$, on $[0,300]$.

Notice that the value of $\mathcal{R}_{0,0}$ in Table 3 is in the range of early estimates by [34] [35] [36] 3.0 (1.5 - 4.5), $2.2(1.4-3.9)$ and 2.68 (2.47 - 2.86), respectively. 
However, there are larger estimates, for instance in [37], $\mathcal{R}_{0} \approx 5.7 \quad(3.8-8.9)$. Moreover, for this time period, there is no $\mathcal{R}_{0}$ estimate available from the $\mathrm{DoH}$ [33].

\subsection{Dynamics of First Wave of the Pandemic}

The first wave of the epidemic in NI is captured in the first 6 interval ( 175 days) of the outbreak. Components $E_{i}, I_{i}$ and $R_{i}$ of the numerical solutions of (2) for $i=0, \cdots, 5$, with initial conditions $\left(S_{i}^{0}, E_{i}^{0}, I_{i}^{0}, R_{i}^{0}, I_{c, i}^{0}\right)=\left(N-\tilde{E}_{0}^{0}, \tilde{E}_{0}^{0}, 0,0,0\right), i=0$ and (3) when $i>0$, are presented in Figure 7 and Figure 8. Between Day 30 and Day 140, $E_{i}$ is mainly decreasing and stays below its value on Day 29, $E_{i}(29)$. Similarly, $I_{i}$ starts to decrease from Day 30, but, in contrast to, $E_{i}$ starts to increase from day 117, and displaying a rapid growth from day 146. These changes in the course of the epidemic most probably related to the UK nationwide interventions introduced on Day 27 (27 $7^{\text {th }}$ March 2020) and gradually relaxed over the summer of 2020. Our numerical solutions suggest that these measures were very effective in slowing down the spread of the disease. Furthermore, our solutions indicate that the number of exposed and infectious individuals started to increase sharply around day 145 (27 $7^{\text {th }}$ July 2020$)$, i.e., about two months after the lockdown measures were eased in NI. During this period of 175 days, $\mathcal{R}_{0, i}<1$, as shown in Table 3 for the rows $i=1,2,3,4$ (days 30 - 146). On the other hand, $\mathcal{R}_{t}^{D}<1$ for days 94 - 122, as shown in Table 4. Finally, notice that $\left.\mathcal{R}_{0,5}=2.9742\left(>\mathcal{R}_{0,0}=2.9539\right)\right)$ is significantly larger than the ones given for the corresponding period in Table 4.

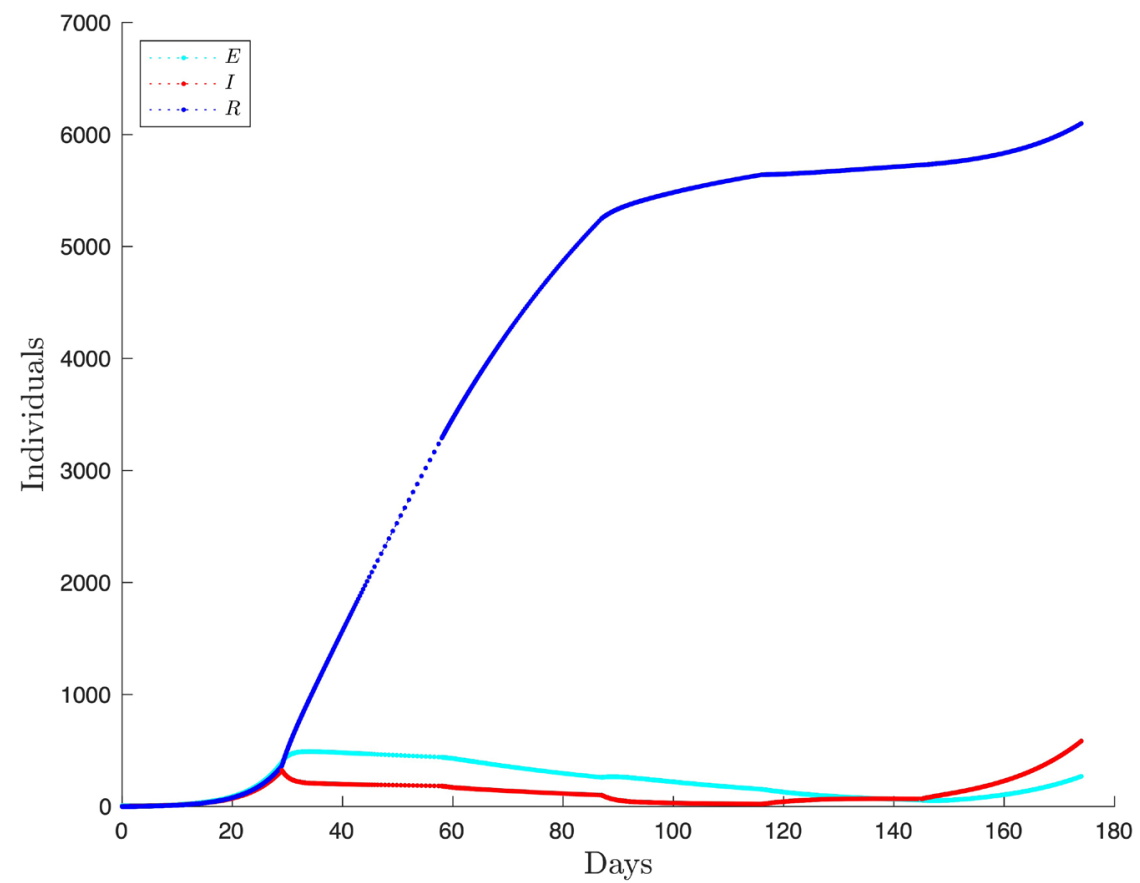

Figure 7. Compartments $E_{i}, I_{i}$ and $R_{i}$ of the numerical solutions of (2) for $i=0, \cdots, 5$ with initial conditions $\left(S_{0}^{0}, E_{0}^{0}, I_{0}^{0}, R_{0}^{0}, I_{c, 0}^{0}\right)=\left(N-\tilde{E}_{0}^{0}, \tilde{E}_{0}^{0}, 0,0,0\right), i=0$ and (3) when $i>0$. 


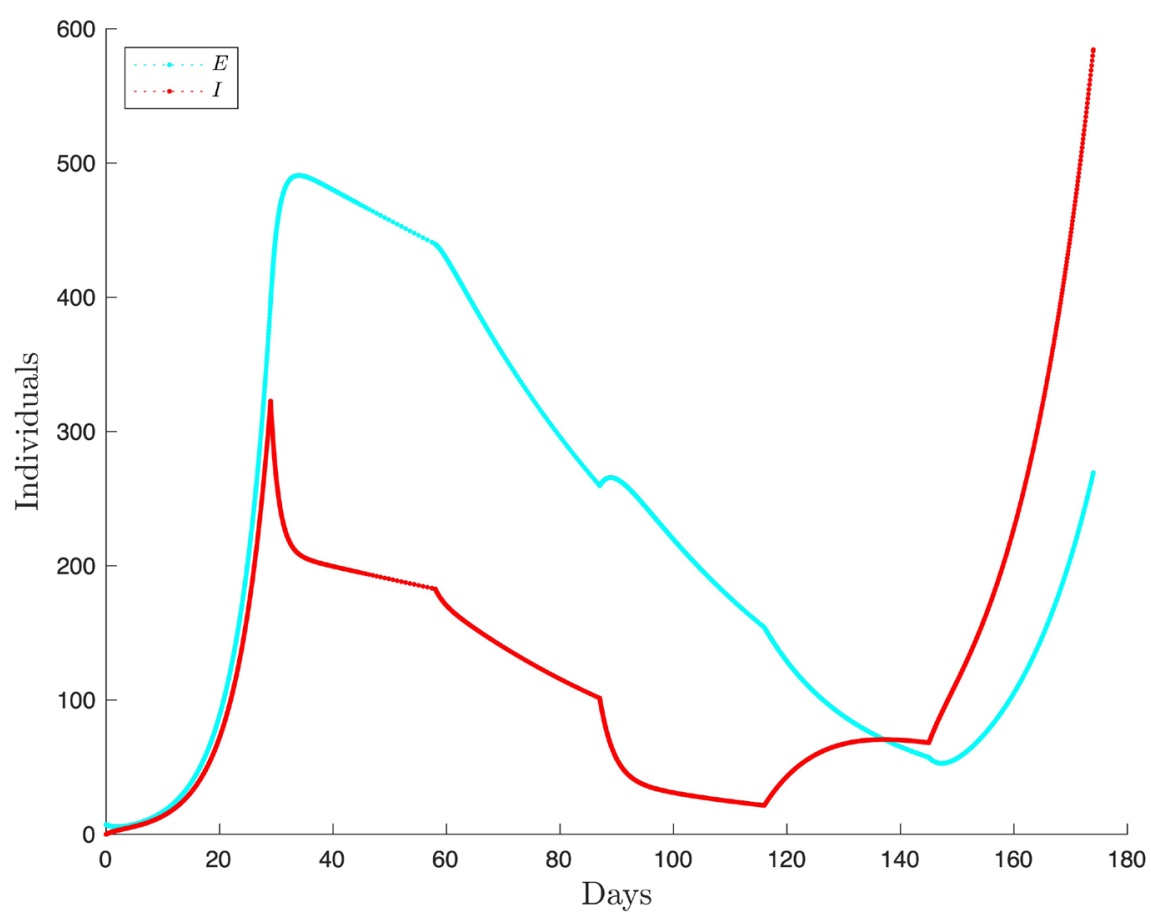

Figure 8. Compartments $E_{i}$ and $I_{i}$ of the numerical solutions of (2) for $i=0, \cdots, 5$ with initial conditions $\left(S_{0}^{0}, E_{0}^{0}, I_{0}^{0}, R_{0}^{0}, I_{c, 0}^{0}\right)=\left(N-\tilde{E}_{0}^{0}, \tilde{E}_{0}^{0}, 0,0,0\right), i=0$ and (3) when $i>0$.

Table 4. Weekly estimates of $\mathcal{R}_{t}^{D}$ for the period of study, provided by the Department of Health of Northern Ireland. [33].

\begin{tabular}{lcc}
\hline Day & Date & $\mathcal{R}_{t}^{D}$ \\
\hline 87 & $26-05-2020$ & $0.8-1.0$ \\
94 & $02-06-2020$ & $0.7-0.9$ \\
101 & $09-06-2020$ & $0.5-0.9$ \\
108 & $16-06-2020$ & $0.6-0.9$ \\
115 & $23-06-2020$ & $0.6-0.9$ \\
122 & $30-06-2020$ & $0.3-0.8$ \\
129 & $07-07-2020$ & $0.5-1.0$ \\
136 & $14-07-2020$ & $0.5-1.0$ \\
143 & $21-07-2020$ & $0.9-1.5$ \\
150 & $28-07-2020$ & $0.5-1.0$ \\
157 & $04-08-2020$ & $1.2-1.4$ \\
164 & $11-08-2020$ & $1.5-1.7$ \\
171 & $18-08-2020$ & $1.2-1.6$ \\
178 & $25-08-2020$ & $1.2-1.6$ \\
185 & $01-09-2020$ & $1.1-1.4$ \\
192 & $08-09-2020$ & $1.1-1.4$ \\
201 & $17-09-2020$ & $1.0-1.3$ \\
\hline & & \\
\hline
\end{tabular}




\begin{tabular}{ccc} 
Continued & & \\
\hline 206 & $22-09-2020$ & $1.4-1.8$ \\
213 & $29-09-2020$ & $1.2-1.6$ \\
220 & $06-10-2020$ & $1.3-1.8$ \\
227 & $13-10-2020$ & $1.4-1.8$ \\
234 & $20-10-2020$ & $1.0-1.3$ \\
241 & $27-10-2020$ & $0.8-1.1$ \\
248 & $03-11-2020$ & $0.6-0.9$ \\
255 & $10-11-2020$ & $0.7-0.95$ \\
262 & $17-11-2020$ & $0.9-1.1$ \\
269 & $24-11-2020$ & $0.75-0.95$ \\
276 & $01-12-2020$ & $0.9-1.1$ \\
283 & $08-12-2020$ & $0.9-1.1$ \\
290 & $15-12-2020$ & $1.0-1.2$ \\
297 & $22-12-2020$ & $1.05-1.25$ \\
\hline
\end{tabular}

\subsection{Dynamics of the Epidemic during the Rest 2020}

We now focus on the later phase of the epidemic. In Table 3, we provide the fitted parameter values and the estimates of $\mathcal{R}_{0, i}$ for the period of study, whereas for completeness, Table 4 presents the estimates of $\mathcal{R}_{t}^{D}$ provided by the $\mathrm{DoH}$ of NI. To obtain numerical solutions of the model (2), we use the initial conditions: $\left(S_{0}^{0}, E_{0}^{0}, I_{0}^{0}, R_{0}^{0}, I_{c, 0}^{0}\right)=\left(N-\tilde{E}_{0}^{0}, \tilde{E}_{0}^{0}, 0,0,0\right)$ for $i=0$, and (3) for $i>0$. The numerical solutions of the $I_{i, c}$ component of the model along with the cumulative number of positives cases, $D_{i}$, for $i=0, \cdots, 9$, are presented in Figure 9. The dynamics of the components $E_{i}$ and $I_{i}$ of the numerical solutions of (2) for $i=0, \cdots, 9$ with initial conditions $\left(S_{0}^{0}, E_{0}^{0}, I_{0}^{0}, R_{0}^{0}, I_{c, 0}^{0}\right)=\left(N-\tilde{E}_{0}^{0}, \tilde{E}_{0}^{0}, 0,0,0\right)$ for $i=0$, and (3) for $i>0$ are presented in Figure 10. The three components $E_{i}, I_{i}$ and $R_{i}$ of the corresponding numerical solutions, are then depicted together in Figure 11, whereas the estimates of $\mathcal{R}_{t}$ are shown in Figure 12.

\section{Discussion and Future Work}

The mathematical modelling of infectious disease spread can have significant utility in providing information and insights that can, if used carefully, facilitate decision making, the implementation of public health strategies as well as allow the use of scarce health care resources, [38] [39]. Criticism of modelling has been levelled, [40], but usually by those who have misunderstood the limitations of modelling: their value is not in long-term-prediction but rather for outlining potential near-future scenarios and how they may be altered by interventions. Clearly to be effective models need to be robust and tested against real word data. Moreover, it has become clear that the multidimensional differences between populations (economic, social, cultural, demographic etc) profoundly influence epidemic dynamics [16] [17]. Hence it is crucial, as we have done, to model in 


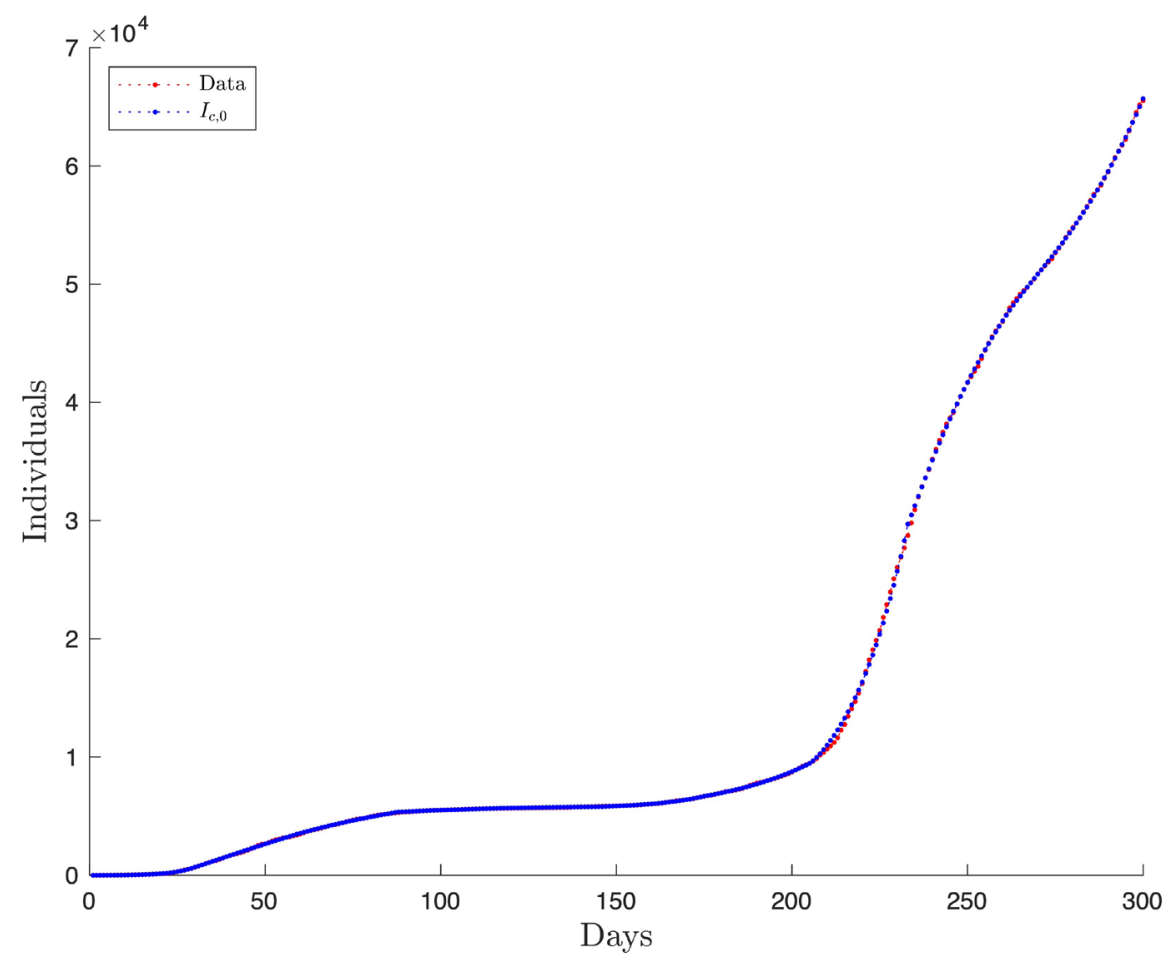

Figure 9. The blue dots represent the $I_{c, 0}$ component of the numerical solution of (2) with $\left(S_{0}^{0}, E_{0}^{0}, I_{0}^{0}, R_{0}^{0}, I_{c, 0}^{0}\right)=\left(N-\tilde{E}_{0}^{0}, \tilde{E}_{0}^{0}, 0,0,0\right)$ and parameters from Table 3 ; the red dots are values of $D_{i}, \quad i=0, \cdots, 9$.

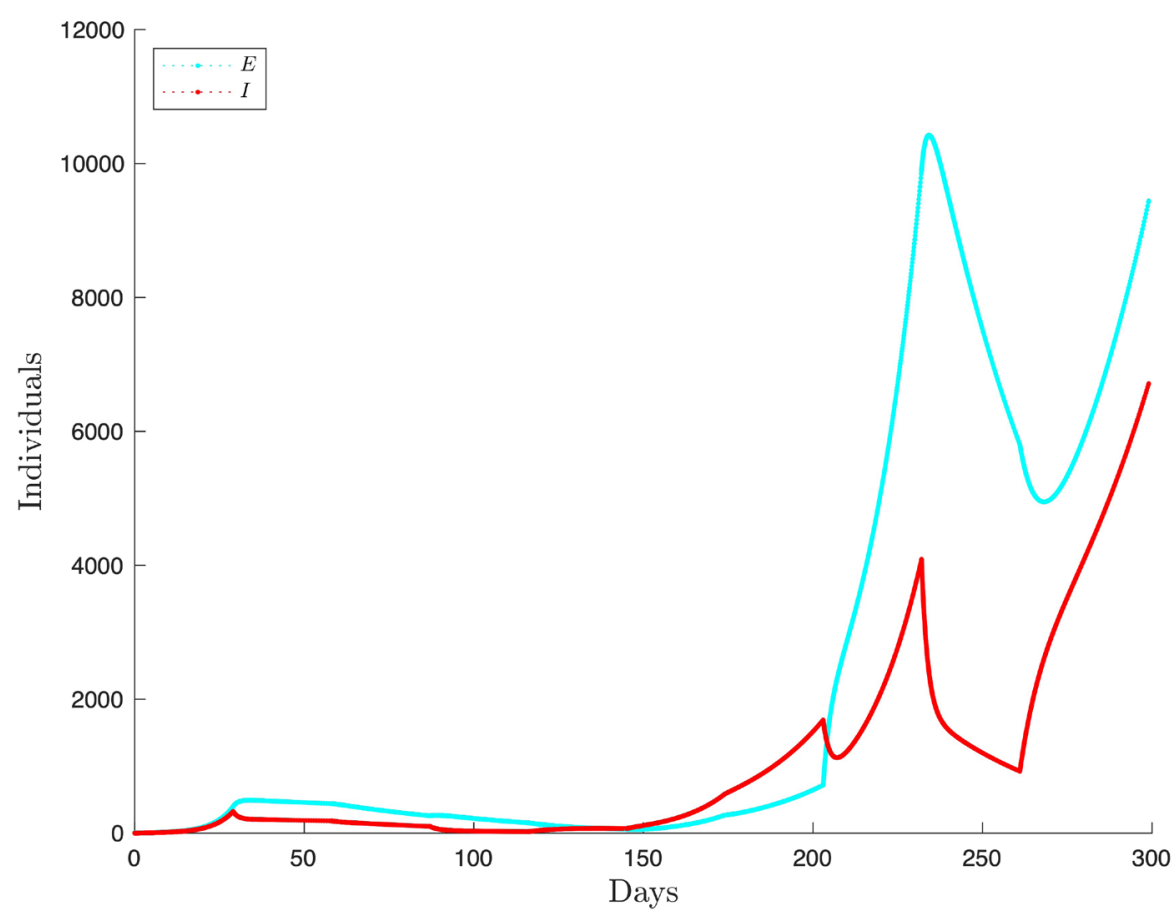

Figure 10. The $E_{i}$ and $I_{i}$ components of the numerical solution of (2) with $\left(S_{0}^{0}, E_{0}^{0}, I_{0}^{0}, R_{0}^{0}, I_{c, 0}^{0}\right)=\left(N-\tilde{E}_{0}^{0}, \tilde{E}_{0}^{0}, 0,0,0\right)$ and parameters from Table 3 and dotted curve is $D_{0}, \quad i=0, \cdots, 9$. 


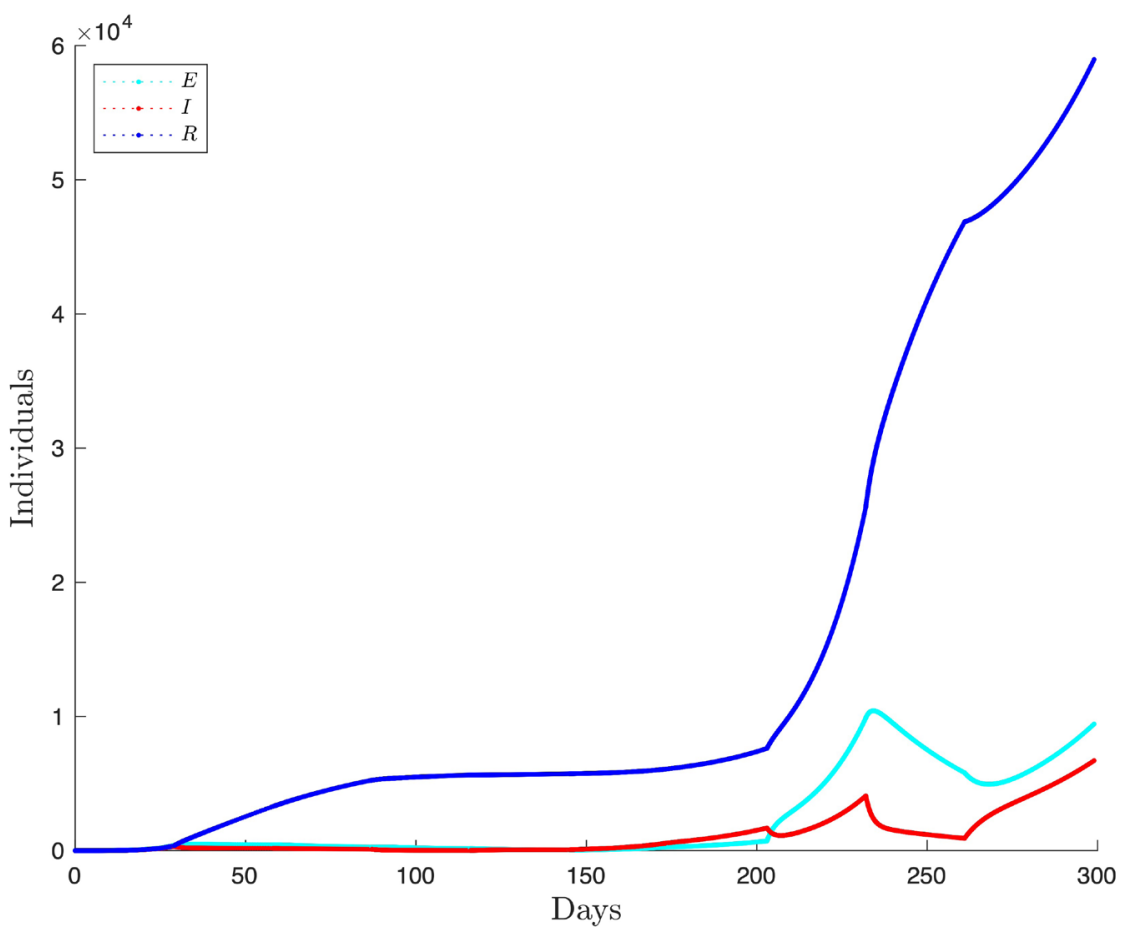

Figure 11. The $E_{i}, I_{i}$ and $R_{i}$ components of the numerical solution of (2) with $\left(S_{0}^{0}, E_{0}^{0}, I_{0}^{0}, R_{0}^{0}, I_{c, 0}^{0}\right)=\left(N-\tilde{E}_{0}^{0}, \tilde{E}_{0}^{0}, 0,0,0\right)$ and parameters from Table 3 , for $i=0, \cdots, 9$.

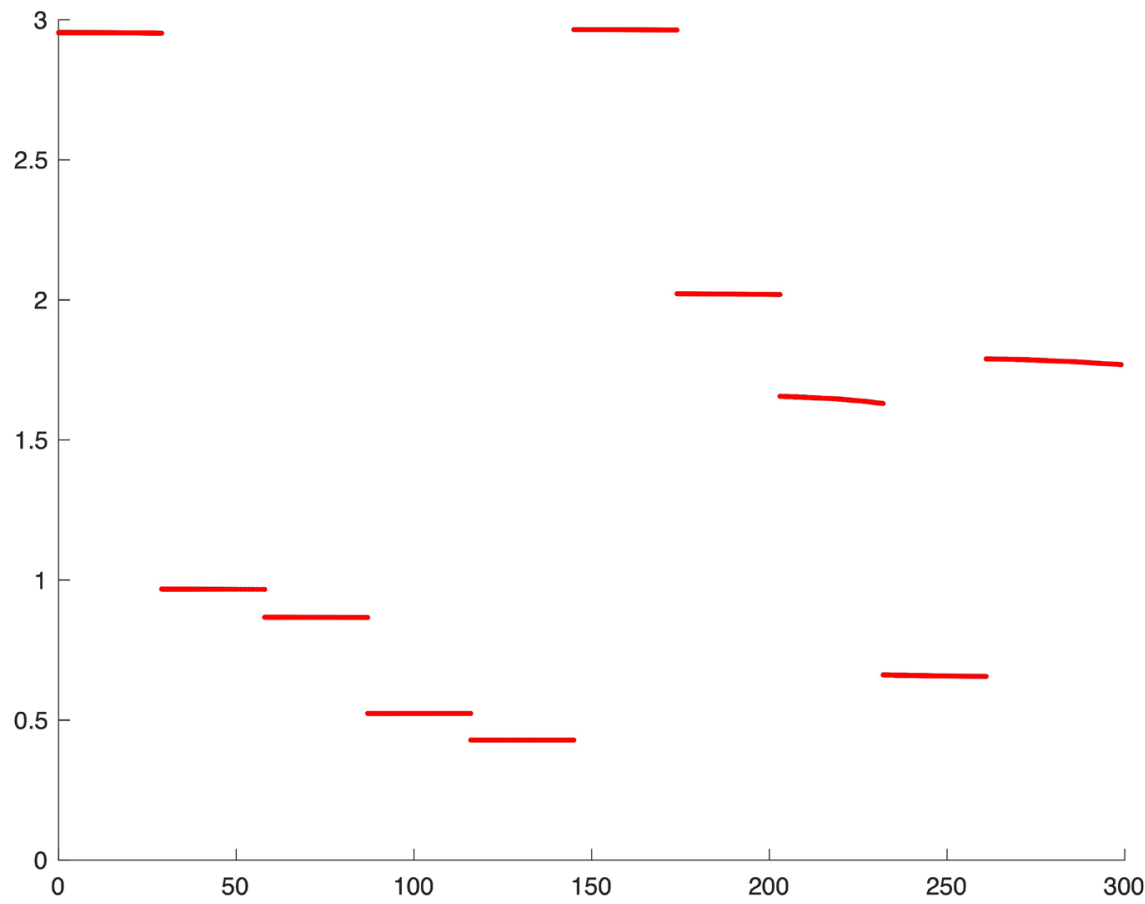

Figure 12. $\mathcal{R}_{t}$ using the estimates in Table 3.

local settings. Northern Ireland is lucky in having a well-developed and robust system for data capture that we have taken advantage of. Using this we successfully fitted SEIR models without vital dynamics to COVID 19 data spanning the 
first two waves of the epidemic. More specifically, we split the data into 10 time intervals, $T_{i}, i=0, \cdots, 9$, and fitted the models to the cumulative number of confirmed positive cases on each interval, and the estimated parameters reflect changes in the level of lockdown measures.

Our study clearly highlights the power of mathematical modelling of epidemiological processes. For instance, by fitting only one variable, $I_{c, i}$, of a sequence of a relatively simple SEIR models to real data, we obtained information about specific features of the disease. For example, by using Table 3, we can estimate that the average incubation time is between 3.4459 and 20 days and that the average recovery time is between 2 and 20 days. However, it is important to note that the mentioned extrema of these estimates, in particular for the infectious period, are the results of the enforced parameter bounds during the fitting process. Nevertheless, the values from these intervals are in good agreement with the widely accepted clinical properties of COVID-19 [34] [35] [36]. In addition, with our modelling approach, assuming only around 7 exposed but no symptomatic individuals at the beginning of the outbreak and clearly importing the disease, we were able to successfully reproduce numerically the evolution of the cumulative number of confirmed positive cases. This fact highlights the necessity of considering strict border control strategies in the case of an emerging pandemic [41], and the crucial role of effective contract tracing methods. Furthermore, the modelling process gives information about variables, the level of exposed and infectious subpopulations at any given time in our case, which are not or partially accessible by current testing methods and strategies. For instance, despite the decreasing number of exposed and infectious individuals, our solutions estimates that, when the easing of the lockdown restrictions was announced, around day 80, the number of exposed and infectious individuals were about 260 and 101 respectively. Although, our computed $\mathcal{R}_{0, i}$ for that and the following period, in agreement with $\mathcal{R}_{t}^{D}$, is below 1 and shows decreasing tendency, shortly after the number of infectious individuals took an upward turn. This might suggest that the reproduction numbers on their own are not sufficient to assess the state of an ongoing pandemic, but in addition, one may wish to consider at least estimates of the number of active infectious individuals in the population.

The vaccination campaign has already been started in NI on $8^{\text {th }}$ December 2020. When data for level of vaccination become available, we aim to update our model to investigate the effects of the campaign on the dynamics of the outbreak in NI. The minimum level of vaccination, with vaccine giving $100 \%$ immunity, to achieve herd immunity is

$$
h_{c}=1-\frac{1}{\mathcal{R}_{0}},
$$

provided $R_{0}>1$, see [42]. Using $\mathcal{R}_{0,0}$ from Table 3, we obtain the following estimate of the herd immunity

$$
h_{c}=1-\frac{1}{\mathcal{R}_{0,0}}=0.6615 .
$$


Notice that

$$
h_{c}^{D}:=\max _{i=0, \cdots, 9}\left(1-\frac{1}{\mathcal{R}_{t}^{D}}\right)=0.4
$$

which is significantly lower than the estimate $(70 \%-80 \%)$ recently reported in the news, [43]

As we mentioned in Section 2, there are many aspects of the disease one may wish to address, such as the level asymptomatic infective subpopulation that we currently do not capture. However, the employed model is not designed to capture this variable. In addition, the SEIR model does not reflect on the finer details of society and interactions therein. For instance, one can obtain a better picture of the disease spread by incorporating the age structure of the considered population and the various levels of interaction between the age groups. Also, during lockdown, various limitations on social interactions were introduced, and possible hubs of disease spread were retail units of essential items. To assess the effectiveness of interventions in this and similar sectors, one can build a model reflecting the features of relatively short, indoor mixings of various subpopulations. In addition, in the case of a developing and ongoing epidemic, the economic impact of imposed control measures should be considered. Metapopulation models considering the spatial distribution and movement patterns within the population can be powerful tools in designing, implementing and updating movement restricting interventions affecting commuter networks vital to maintaining a healthy economy even in the case of an epidemic. Furthermore, during the last week of December, the level of the rates of infection is less likely to be captured by the data due to changes in behaviour that can affect testing. In the week up to $4^{\text {th }}$ January, there was a steep increase in the reported positive tests, which could result in increased hospital admissions. There was a steady increase in the reported positive cases despite the restriction measures introduced by the local authorities on $26^{\text {th }}$ December 2020 to control the spread of the disease. This surge in the daily incidence of infection may be attributed to the new strain of COVID-19 also known as Variant of Concern (SARS-CoV-2 lineage B.1.1.7), detected in November 2020, or socializing or both. SARS-CoV-2 lineage B.1.1.7 is rapidly spreading across England as well as the regions of the UK. In a recent study [44], the authors evaluated the relationship between the transmission and the frequency of SARS-CoV-2 lineage B.1.1.7 across regions in England over time and found a higher transmissibility compared to SARS-CoV-2. Modifications of the current model will address this as data become available.

Despite its limitation, our model robustly captures the essential elements of the first 12 months of the NI COVID-19 epidemic and is a platform for further development. Developing further our model by considering additional features of the COVID-19 pandemic and the refined details of the population in NI, we aim at improving the quality of the parameter estimates and the details of information offered by compartmental modelling of the spread of communicable diseases. 


\section{Conclusion}

We successfully fitted several copies of a Susceptible-Exposed-Infectious-Recovered (SEIR) compartmental model on consecutive time intervals between $1^{\text {st }}$ March 2020 and $25^{\text {th }}$ December 2020 to the cumulative number of confirmed positive cases of COVID-19 in Northern Ireland. We provided estimates of the basic reproduction number and the effective reproduction number. In addition, based on our parameter estimates, we discussed the required level of herd immunity to disrupt the chain of COVID-19 infection in Northern Ireland.

\section{Conflicts of Interest}

The authors declare no conflicts of interest regarding the publication of this paper.

\section{References}

[1] World Health Organization (2020) Coronavirus Disease 2019 (COVID-19) Situation Report 11.

[2] World Health Organization (2020) Coronavirus Disease 2019 (COVID-19) Situation Report 51.

[3] World Health Organization (2020) Coronavirus Disease (COVID-19) Pandemic. Numbers at a Glance.

https://www.who.int/emergencies/diseases/novel-coronavirus-2019

[4] Bertozzi, A.L., Franco, E., Mohler, G., Short, M.B. and Sledge, D. (2020) The Challenges of Modeling and Forecasting the Spread of COVID-19. Proceedings of the National Academy of Sciences of the United States of America, 117, 16732-16738. https://doi.org/10.1073/pnas.2006520117

[5] Lin, Q., Zhao, S., Gao, D., et al. (2020) A Conceptual Model for the Coronavirus Disease 2019 (COVID-19) Outbreak in Wuhan, China with Individual Reaction and Governmental Action. International Journal of Infectious Diseases, 93, 211-216. https://doi.org/10.1016/j.ijid.2020.02.058

[6] IHME COVID-19 Forecasting Team (2020) Modeling COVID-19 Scenarios for the United States. Nature Medicine, 27, 94-105. https://doi.org/10.1038/s41591-020-1132-9

[7] Davies, N.G., Kucharski, A.J., Eggo, R.M., Gimma, A., CMMID COVID-19 Working Group and Edmunds, W.J. (2020) The Effects of Non-Pharmaceutical Interventions on COVID-19 Cases, Deaths, and Demand for Hospital Services in the UK: A Modelling Study. Lancet Public Health, 5, e375-e385. https://doi.org/10.1101/2020.04.01.20049908

[8] Giordano, G., Blanchini, F., Bruno, R., et al. (2020) Modelling the COVID-19 Epidemic and Implementation of Population-Wide Interventions in Italy. Nature Medicine, 26, 855-860. https://doi.org/10.1038/s41591-020-0883-7

[9] Sebastiani, G., Massa, M. and Riboli, E. (2020) COVID-19 Epidemic in Italy: Evolution, Projections and Impact of Government Measures. European Journal of Epidemiology, 35, 341-345. https://doi.org/10.1007/s10654-020-00631-6

[10] Aràndiga, F., Baeza, A., Cordero-Carrión, I., et al. (2020) A Spatial-Temporal Model for the Evolution of the COVID-19 Pandemic in Spain Including Mobility. Mathematics, 8, 1677. https://doi.org/10.3390/math8101677 
[11] Böhmer, M.M., Buchholz, U., Corman, V.M., et al. (2020) Investigation of a COVID-19 Outbreak in Germany Resulting from a Single Travel-Associated Primary Case: A Case Series. Lancet Infectious Disease, 20, 920-928. https://doi.org/10.1016/S1473-3099(20)30314-5

[12] Hoertel, N., Blachier, M., Blanco, C., et al. (2020) A Stochastic Agent-Based Model of the SARS-CoV-2 Epidemic in France. Nature Medicine, 26, 1417-1421. https://doi.org/10.1038/s41591-020-1001-6

[13] Humphries, R., Spillane, M., Mulchrone, K., et al. (2020) A Metapopulation Network Model for the Spreading of SARS-CoV-2: Case Study for Ireland. Infectious Disease. https://doi.org/10.1101/2020.06.26.20140590

[14] Röst, G., Bartha, F.A., Bogya, N., et al. (2020) Early Phase of the COVID-19 Outbreak in Hungary and Post-Lockdown Scenarios. Viruses, 17, 708.

https://doi.org/10.3390/v12070708

[15] Azanza, C. and Hernandez-Vargas, E.A. (2020) Epidemiological Characteristics of COVID-19 in Mexico and the Potential Impact of Lifting Confinement across Regions. Frontiers in Physics, 8, 458. https://doi.org/10.3389/fphy.2020.573322

[16] Eggo, R.M., Dawa, J., Kucharski, A.J. and Cucunuba, Z.M. (2021) The Importance of Local Context in COVID-19 Models. Nature Computational Science, 1, 6-8. https://doi.org/10.1038/s43588-020-00014-7

[17] Rhodes, T., Lancaster, K., Lees, S. and Parker, M. (2020) Modelling the Pandemic: Attuning Models to Their Contexts. BMJ Global Health, 5, e002914.

https://doi.org/10.1136/bmjgh-2020-002914

[18] Department of Health-Northern Ireland (2020) COVID-19 Daily Dashboard Updates. https://www.health-ni.gov.uk/articles/covid-19-daily-dashboard-updates

[19] Northern Ireland Statistics and Research Agency. 2019 Mid Year Population Estimates for Northern Ireland.

https://www.nisra.gov.uk/sites/nisra.gov.uk/files/publications/MYE19-Bulletin.pdf

[20] Brauer, F. and Castillo-Chavez, C. (2012) Mathematical Models in Population Biology and Epidemiology, Volume 2. Springer, Berlin. https://doi.org/10.1007/978-1-4614-1686-9

[21] Brauer, F., den Driessche, P.V. and Wu, J. (2008) Lecture Notes in Mathematical Epidemiology. Springer, Berlin. https://doi.org/10.1007/978-3-540-78911-6

[22] Kermack, W.O. and McKendrick, A.G. (1927) A Contribution to the Mathematical Theory of Epidemics. Proceedings of the Royal Society of London. Series A, Containing Papers of a Mathematical and Physical Character, 115, 700-721. https://doi.org/10.1098/rspa.1927.0118

[23] Linton, N.M., Kobayashi, T., Yang, Y., Hayashi, K., Akhmetzhanov, A.R., Jung, S.-M., Yuan, B., Kinoshita, R. and Nishiura, H. (2020) Incubation Period and Other Epidemiological Characteristics of 2019 Novel Coronavirus Infections with Right Truncation: A Statistical Analysis of Publicly Available Case Data. Journal of Clinical Medicine, 9, 538. https://doi.org/10.3390/jcm9020538

[24] Wang, Y., Wang, Y., Chen, Y. and Qin, Q. (2020) Unique Epidemiological and Clinical Features of the Emerging 2019 Novel Coronavirus Pneumonia (COVID-19) Implicate Special Control Measures. Journal of Medical Virology, 92, 568-576. https://doi.org/10.1002/jmv.25748

[25] Barbarossa, M.V., Dénes, A., Kiss, G., Nakata, Y., Röst, G. and Vizi, Z. (2015) Transmission Dynamics and Final Epidemic Size of Ebola Virus Disease Outbreaks with Varying Interventions. PLoS ONE, 10, e0131398.

https://doi.org/10.1371/journal.pone.0131398 
[26] Lekone, P.E. and Finkenstädt, B.F. (2006) Statistical Inference in a Stochastic Epidemic SEIR Model with Control Intervention: Ebola as a Case Study. Biometrics, 62, 1170-1177. https://doi.org/10.1111/j.1541-0420.2006.00609.x

[27] Cintrón-Arias, A., Castillo-Chávez, C., Bettencourt, L.M.A., Lloyd, A.L. and Banks, H.T. (2009) The Estimation of the Effective Reproductive Number from Disease Outbreak Data. Mathematical Biosciences and Engineering, 6, 261-282. https://doi.org/10.3934/mbe.2009.6.261

[28] Li, M.Y. and Muldowney, J.S. (1995) Global Stability for the SEIR Model in Epidemiology. Mathematical Biosciences, 125, 155-164. https://doi.org/10.1016/0025-5564(95)92756-5

[29] He, X., Lau, E.H., Wu, P., Deng, X., Wang, J., Hao, X., Lau, Y.C., Wong, J.Y., Guan, Y., Tan, X., et al. (2020) Temporal Dynamics in Viral Shedding and Transmissibility of COVID-19. Nature Medicine, 26, 672-675. https://doi.org/10.1038/s41591-020-0869-5

[30] Lauer, S.A., Grantz, K.H., Bi, Q., Jones, F.K., Zheng, Q., Meredith, H.R., Azman, A.S., Reich, N.G. and Lessler, J. (2020) The Incubation Period of Coronavirus Disease 2019 (COVID-19) from Publicly Reported Confirmed Cases: Estimation and Application. Annals of Internal Medicine, 172, 577-582.

https://doi.org/10.7326/M20-0504

[31] World Health Organization (2020) Coronavirus Disease 2019 (COVID-19) Situation Report 73.

[32] MATLAB (2020) Version 9.8.0.1417392 (R2020a). The MathWorks Inc., Natick.

[33] Department of Health (2020) R Number Papers. https://www.health-ni.gov.uk/R-Number. Last accessed on 08/01/2021

[34] Kucharski, A.J., Russell, T.W., Diamond, C., Liu, Y., Edmunds, J., Funk, S. and Eggo, R.M. (2020) Early Dynamics of Transmission and Control of COVID-19: A Mathematical Modelling Study. The Lancet Infectious Diseases, 20, 553-558. https://doi.org/10.1016/S1473-3099(20)30144-4

[35] Li, Q., Guan, X., Wu, P., Wang, X., Zhou, L., Tong, Y., Ren, R., Leung, K.S., Lau, E.H., Wong, J.Y., et al. (2020) Early Transmission Dynamics in Wuhan, China, of Novel Coronavirus-Infected Pneumonia. New England Journal of Medicine, 382, 1199-1207.

[36] Wu, J.T., Leung, K. and Leung, G.M. (2020) Nowcasting and Forecasting the Potential Domestic and International Spread of the 2019-nCov Outbreak Originating in Wuhan, China: A Modelling Study. The Lancet, 395, 689-697. https://doi.org/10.1016/S0140-6736(20)30260-9

[37] Sanche, S., Lin, Y.T., Xu, C., et al. (2020) High Contagiousness and Rapid Spread of Severe Acute Respiratory Syndrome Coronavirus 2. Emerging Infectious Disease, 26, 1470-1477. https://doi.org/10.3201/eid2607.200282

[38] Friston, K.J., Costello, A. and Pillay, D. (2020) “Dark Matter", Second Waves and Epidemiological Modelling. BMJ Global Health, 5, e003978.

https://doi.org/10.1101/2020.09.01.20185876

[39] Sun, K., Wang, W., Gao, L., Wang, Y., Luo, K., Ren, L., Zhan, Z., Chen, X., Zhao, S., Huang, Y., et al. (2021) Transmission Heterogeneities, Kinetics, and Controllability of SARS-CoV-2. Science, 371, eabe2424. https://doi.org/10.1126/science.abe2424

[40] Holmdahl, I. and Buckee, C. (2020) Wrong but Useful-What COVID-19 Epidemiologic Models Can and Cannot Tell Us. New England Journal of Medicine, 383, 303-305. https://doi.org/10.1056/NEJMp2016822

[41] Stuff (2021) Coronavirus: Government Shuts Borders to All but Citizens and Resi- 
dents.

https://www.stuff.co.nz/national/health/coronavirus/120423903/coronavirus-primeminister-to-make-announcement-on-border-controls

[42] Britton, T., Ball, F. and Trapman, P. (2020) A Mathematical Model Reveals the Influence of Population Heterogeneity on Herd Immunity to SARS-CoV-2. Science, 369, 846-849. https://doi.org/10.1126/science.abc6810

[43] BBC (2021) Coronavirus: Lifting NI Restrictions Will “Need 70-80\% Vaccinated”. https://www.bbc.co.uk/news/uk-northern-ireland-56000840

[44] Volz, E., Mishra, S., Chand, M., Barrett, J.C., et al. (2020) Report 42-Transmission of SARS-CoV-2 Lineage B.1.1.7 in England: Insights from Linking Epidemiological and Genetic Data. MRC Centre for Global Infectious Disease Analysis-Imperial College, London. https://doi.org/10.1101/2020.12.30.20249034 